\title{
Mesenchymal stem cells protect podocytes from apoptosis induced by high glucose via secretion of epithelial growth factor
}

\author{
Diangeng $\mathrm{Li}^{1+}$, Nan Wang ${ }^{1 \dagger}$, Li Zhang ${ }^{1}$, Zhu Hanyu${ }^{1}$, Bai Xueyuan ${ }^{1}, \mathrm{Bo} \mathrm{Fu}{ }^{1}$, Cui Shaoyuan ${ }^{1}$, Weiguang Zhang ${ }^{1}$, \\ Sun Xuefeng ${ }^{1}$, Rongshan $\mathrm{Li}^{2^{*}}$ and Xiangmei Chen ${ }^{1^{*}}$
}

See related commentary by Ali and Brazil, http://stemcellres.com/content/4/5/119

\begin{abstract}
Introduction: The apoptosis and subsequent injury of podocytes plays a pathogenic role in diabetic nephropathy (DN). Mesenchymal stem cells (MSCs) are promising therapeutic cells for preventing apoptosis and reducing cellular injury. Our previous study found that MSCs could protect kidneys from diabetes-induced injury without obvious engraftment. So we evaluated the effects of human adipose-derived MSCs (hAd-MSCs) on podocytic apoptosis and injury induced by high glucose $(\mathrm{HG})$ and the underlying mechanisms.

Methods: We used flow cytometry, Western blot and confocal fluorescence microscopy to study podocytic apoptosis and injury induced by HG at 24 hours, 48 hours, and 72 hours in the presence or absence of MSC-conditioned medium (CM). An antibody-based cytokine array was used to identify the mediating factor, which was verified by adding the neutralizing antibody (NtAb) to block its function or adding the recombinant cytokine to the medium to induce its function.

Results: hAd-MSC-CM reduced podocytic apoptosis in a dose-dependent manner, decreased the expression of podocytic cleaved caspase-3, and prevented the reduced expression and maintained the normal arrangement of podocytic synaptopodin and nephrin. However, human embryonic lung cell (Wi38)-CM failed to ameliorate podocytic apoptosis or injury. Twelve cytokines with concentration ratios (MSC-CMNi38-CM) >10-fold were identified. Epithelial growth factor (EGF) was singled out for its known ability to prevent apoptosis. Recombinant human EGF (rhEGF) prevented podocytic apoptosis and injury similarly to hAd-MSC-CM but, upon blockade of EGF, the beneficial effect of hAd-MSC-CM decreased dramatically.
\end{abstract}

Conclusions: hAd-MSCs prevent podocytic apoptosis and injury induced by HG, mainly through secreting soluble EG.

Keywords: Apoptosis, Diabetic nephropathy, Epithelial growth factor, Injury, Mesenchymal stem cells, Podocyte

\section{Introduction}

Diabetic nephropathy (DN) is one of the most common and serious complications of capillaries in patients with diabetes [1]. In recent years, research on the structure and function of the filtration barrier of the glomerulus has focused on the function of podocytes in the

\footnotetext{
* Correspondence: rongshanli@126.com; xmchen301@126.com ${ }^{\dagger}$ Equal contributors

${ }^{2}$ Department of Nephrology, The Second Hospital of Shanxi Medical University, 382\# Wu Yi Road, Tai Yuan, Shanxi 030001, China 'Department of Nephrology, State Key Laboratory of Kidney Diseases, Chinese PLA General Hospital and Medical School of Chinese PLA, 28\# Fu Xing Road, Beijing 100853, China
}

occurrence and development of DN [2]. Podocytes are terminally differentiated epithelial cells attached to the glomerular basement membrane, and the alternative podocytic processes between adjacent podocytes construct the slit diaphragm, which plays an important role in maintaining the integrity of the filtration barrier structure and function of the glomerulus [3-5]. High glucose contributes to reduced podocyte number and induces apoptosis of cultured podoctes and causes albuminuria and accelerates foot process effacement $[2,3,6,7]$. At present, there is no satisfactory method or target for the treatment of $\mathrm{DN}$.
C Biomed Central

(c) 2013 Li et al.; licensee BioMed Central Ltd. This is an Open Access article distributed under the terms of the Creative Commons Attribution License (http://creativecommons.org/licenses/by/2.0), which permits unrestricted use, distribution, and reproduction in any medium, provided the original work is properly cited. 
Mesenchymal stem cells (MSCs), a group of cells from the mesoblast that have characteristics of stem cells, show strong proliferation ability and multipotential differentiation, which can repair injured tissues and cells by secreting cytokines [8-10]. Treatments involving MSCs may be effective for repairing injured tissues and cells clinically $[8,11]$. It has been shown that MSCs can promote tissue repair through chemotaxis and homing to the damaged location in vivo, or through the paracrine action of various cytokines, to reduce adverse reactions of the kidney, so as to improve and promote the endogenous repair of kidney tissue [12-15]. It is easy to obtain MSCs from adipose tissue, which has stable cellularity. After high passage of these cells, only a low level of senescence will occur [16]. These cells have the ability to secrete a large number of protective cytokines $[17,18]$ and show other MSCs characteristics, such as self-renewal and multiple lineage differentiation. In preliminary experiments, we found that human adiposederived (hAd)-MSCs injected via the tail vein into rats with DN alleviated kidney injury, reduced proteinurea, and prevented the downregulation of synaptopodin (data not shown); however, the gross presence of stem cells was not found in the kidney. Thus, we speculated that hAd-MSCs protected the kidney via paracrine action.

Using hAd-MSC-conditioned medium (CM) with a model of podocytic apoptosis induced by high glucose (HG), we investigated whether MSCs-CM could itself inhibit HG-induced podocytic apoptosis, to identify the effect or molecule(s) and to provide novel insights into the treatment of DN.

\section{Methods}

\section{Cell culture and processing}

A mouse podocyte clone 5 (MPC5), which was provided by Professor Peter Mundel of the Medical College of Harvard University (Boston, MA, USA), was cultured as previously described $[16,19]$. Briefly, cryopreserved podocytes were cultured and amplified in $11 \mathrm{mM} \mathrm{D}$ glucose Roswell Park Memorial Institute (RPMI)-1640 medium containing 20 units $/ \mathrm{ml}$ IFN- $\gamma$ (Invitrogen, Carlsabad, CA, USA) and $10 \%$ FCS at $33^{\circ} \mathrm{C}$. After passage at $37^{\circ} \mathrm{C}$, the podocytes were cultured in IFN- $\gamma$ free medium for 10 days (changing the solution every two days) to induce differentiation. The presence of synaptopodin and nephrin indicated podocytic differentiation and was detected by confocal microscopy. The cells were cultured synchronously in medium containing $0.2 \%$ FCS and $5.5 \mathrm{mM}$ D-glucose RPMI-1640 medium for 24 hours before the experiment. MPC5 cells were divided mainly into seven groups according to treatment: normal glucose (NG, $5.5 \mathrm{mM})$, mannitol control (NG+Ma, 5.5 mM D-glucose + $24.5 \mathrm{mM}$ mannitol),
HG (30 mM), treatment group in human embryonic lung cells $(\mathrm{Wi} 38)-\mathrm{CM}(1 \times, 2 \times, 4 \times)$, treatment group in MSC-CM $(1 \times, 2 \times, 4 \times)$, recombinant human epidermal growth factor (rhEGF, $3 \mathrm{ng} / \mathrm{ml}$ ) treatment group, and MSC-CM neutralizing antibody (MSC-CM+EGF+NtAb) group.

hAd-MSCs were provided by the Microcirculation Institute of the Chinese Academy of Medical Sciences (Beijing, China). These cells from patients undergoing selective suction-assisted lipectomy were collected after obtaining informed consent from the patients according to procedures approved by the Ethics Committtee of the Chinese Academy of Medical Sciences and Peking Union Medical College. The adipose tissue was extensively washed with D-Hanks' solution to remove contaminating blood cells and local anesthetics. Then, it was resuspended in $0.075 \%$ typeIA collagenase (Sigma-Aldrich Corp, St. Louis, MO, USA)/Hank's Balanced Salt Solution (approximately 2 $\mathrm{ml} / \mathrm{g}$ ) and incubated at $37^{\circ} \mathrm{C}$ for one hour to release the cellular fractions. The digested adipose tissue was passed through a $100-\mu \mathrm{m}$ filter to remove debris and centrifuged at $150 \times \mathrm{g}$ for 10 minutes to produce a cell pellet. After isolation, $30 \mathrm{ml}$ of resuspended cells were plated in expansion medium at a density of 5 to approximately $6 \times 10^{6}$ nucleated cells $/ 75 \mathrm{~cm}^{2}$ tissue culture dish and incubated at $37^{\circ} \mathrm{C}$. Once adherent cells were more than $80 \%$ confluent, they were detached with $0.125 \%$ trypsin and $0.01 \%$ ethylenediaminetetraacetic acid (EDTA) and replated at a 1:4 dilution under the same conditions according to previous protocols $[16,20]$. All the experiments were done with the 5th passage and the 20th passage and the 5th passage of hAd-MSCs was used for the results given in this article.

Wi38 were purchased from the American Type Culture Collection (ATCC, Manassas, VA, USA) and were cultured in RPMI-1640 medium containing 10\% FCS and passaged when confluence reached $80 \%$.

\section{Preparation of CM}

hAd-MSCs and Wi38 cells were cultured in their respective media at a concentration of $1 \times 10^{5}$ cells $/ \mathrm{cm}^{2}$ and were rinsed three times in PBS after one day of cell adhesion. Then, the cells were cultured in serum-free RPMI-1640 medium for 48 hours and the culture supernatants were collected. The supernatants were centrifuged at $1,500 \mathrm{rpm}$ for 10 minutes at $4^{\circ} \mathrm{C}$ and transferred to a centrifugal column with a $3 \mathrm{kDa}$ cut-off (Millipore, Billerica, MA, USA). This resulted in $15 \times$ MSC-CM or Wi38-CM, respectively, which were desalted according to the manufacturer's protocol. CM was then sterilized by filtration through a $220 \mathrm{~nm}$ filter (Millipore, Billerica, MA, USA). 


\section{The expression of cleaved caspase- 3 in MPC5 cells detected by Western blot}

MPC5 cells were collected, and the total protein was extracted. The protein concentration was determined by BCA assay (Pierce, Rockford, IL, USA), and $2 \times$ SDSdenatured protein $\left(95^{\circ} \mathrm{C}\right.$ for 5 minutes and ice-bath for 10 minutes) in the same volume was added. The total protein of podocytes was isolated using 15\% SDS-PAGE and $80 \mu \mathrm{g}$ of each sample. The isolated protein was transferred to a nitrocellulose membrane. The primary rabbit polyclonal anti-cleaved caspase- 3 antibody (Cell Signaling Technology, Beverly, MA, USA) was diluted 1:1000 and incubated with the membrane(s) overnight at $4^{\circ} \mathrm{C}$. Then the secondary anti-rabbit antibody (Santa Cruz Biotechnology, Santa Cruz, CA, USA), conjugated to horseradish peroxidase (HRP) and diluted to 1:1000, was added for one hour at room temperature. $\beta$-actin was used as the loading control and the blots were developed with an ECL Western Blotting kit (Applygen Technologies Inc, Beijing, China).

\section{Podocytic apoptosis detected by flow cytometry}

After the podocytes treated by different methods were cultured at $37^{\circ} \mathrm{C}$ for 24,48 , and 72 hours, respectively, and were digested with $0.25 \%$ EDTA-free pancreatin (Gibco, Grand Island, NY, USA), they were rinsed twice in PBS. The cell concentration was adjusted to $1 \times 10^{6}$ cells/L to make a single cell suspension. An annexin V/ propidium iodide (PI) double staining apoptosis detection kit (BD Biosciences, Franklin Lakes, NJ, USA) was used to stain the podocytes according to the manufacturer's instructions. The data were acquired on a flow cytometer (Beckman Coulter Inc, CA, USA).

After the podocytes treated by different methods were cultured at $37^{\circ} \mathrm{C}$ for 24,48 , and 72 hours, respectively, the cell concentration was adjusted to $1 \times 106$ cells/L to make a single cell suspension. When the apoptosis models were established, reflecting the degree of apoptosis, was measured using flow cytometry with TUNEL staining. An In Situ Cell Death Detection Kit (Roche, Mannheim, Germany) was used to stain podocytes according to the manufacturer's instructions. The data were also acquired on a flow cytometer (Beckman Coulter Inc).

\section{Cellular immunofluorescence}

Differentiated and matured podocytes were seeded onto six-pore plates and covered by a cover glass that was pretreated with collagen I (Sigma-Aldrich, St. Louis, MO, USA). Each group was subjected to its own designated treatment regimen, and the cells were cultured at $37^{\circ} \mathrm{C}$ for 24,48 and 72 hours, respectively. Three accessory foramina were set up for each group at each time point. The cells were fixed with $4 \%$ paraformaldehyde for 5 minutes at room temperature followed by incubation at $4^{\circ} \mathrm{C}$ for 10 minutes. Then, $0.2 \%$ Triton X100 was added to permeabilize the cells for 5 minutes at room temperature and $1 \times$ casin was sealed up for $30 \mathrm{mi}$ nutes at room temperature. Primary rabbit polyclonal antibodies specific for synaptopodin and nephrin were diluted 1:50 and incubated with the cells (Santa Cruz Biotechnology) (ProSci Incorporated, San Diego, California, USA) overnight at $4^{\circ} \mathrm{C}$. A secondary anti-rabbit antibody conjugated to $\mathrm{Cy} 3$ (Jackson ImmunoResearch Laboratories, Bar Harbor, ME, USA) (diluted 1:1000) was incubated with the cells away from light for one hour at room temperature. Finally, a fluorescent sealing liquid (ZSGB-BIO, Beijing, China) containing 4',6-diamidino-2phenylindole (DAPI) was added, and confocal laser scanning microscopy (FluoView FV1000; Olympus America Inc., Center Valley, PA, USA) was used to determine the expression level and structure arrangement of podocytic synaptopodin and nephrin. Ten visual fields were observed at random in each culture pore.

\section{Antibody chip}

A cytokine detection kit (RayBio Human Cytokine Array; RayBiotech, Inc., Norcross, GA, USA) was used to determine the presence of cytokines in the $15 \times$ concentrated blank RPMI-1640 medium, Wi38-CM, and Ad-MSCs-CM. The analysis was performed using the manufacturer's recommended protocol, and the signals of $\mathrm{Cy} 3$ were imaged by Axon GenePix laser scanner (MDS Analytical Technologies, Sunnyvale, CA, USA). The result was normalized to the respective positive control. Results were obtained from three independent samples.

\section{Cytokines detected by enzyme-linked immunosorbent assay (ELISA)}

The concentration of EGF, insulin-like growth factor binding protein (IGFBP)-1, glial cell line-derived neurotrophic factor (GDNF) and placental growth factor (PIGF) was determined in each culture supernatant (RPMI-1640 medium, Wi38-CM and Ad-MSC-CM) by ELISA kits (R\&D Systems, Minneapolis, MN, USA) according to the manufacturer's instructions. Optical density (OD) was measured at $450 \mathrm{~nm}$ and $570 \mathrm{~nm}$. Concentration analysis software (Microplate Manager 4.0) was used to calculate the concentration of candidate factors in the samples according to standard curves. Three independent samples were placed in three repetitive holes.

\section{Recombinant factor and $\mathrm{NtAb}$ experiments}

To prepare rhEGF, rhIGFBP-1, rhGDNF and rhPIGF, the respective lyophilized powders (PeproTech, RockyHill, NJ, USA) were reconstituted to a working concentration 


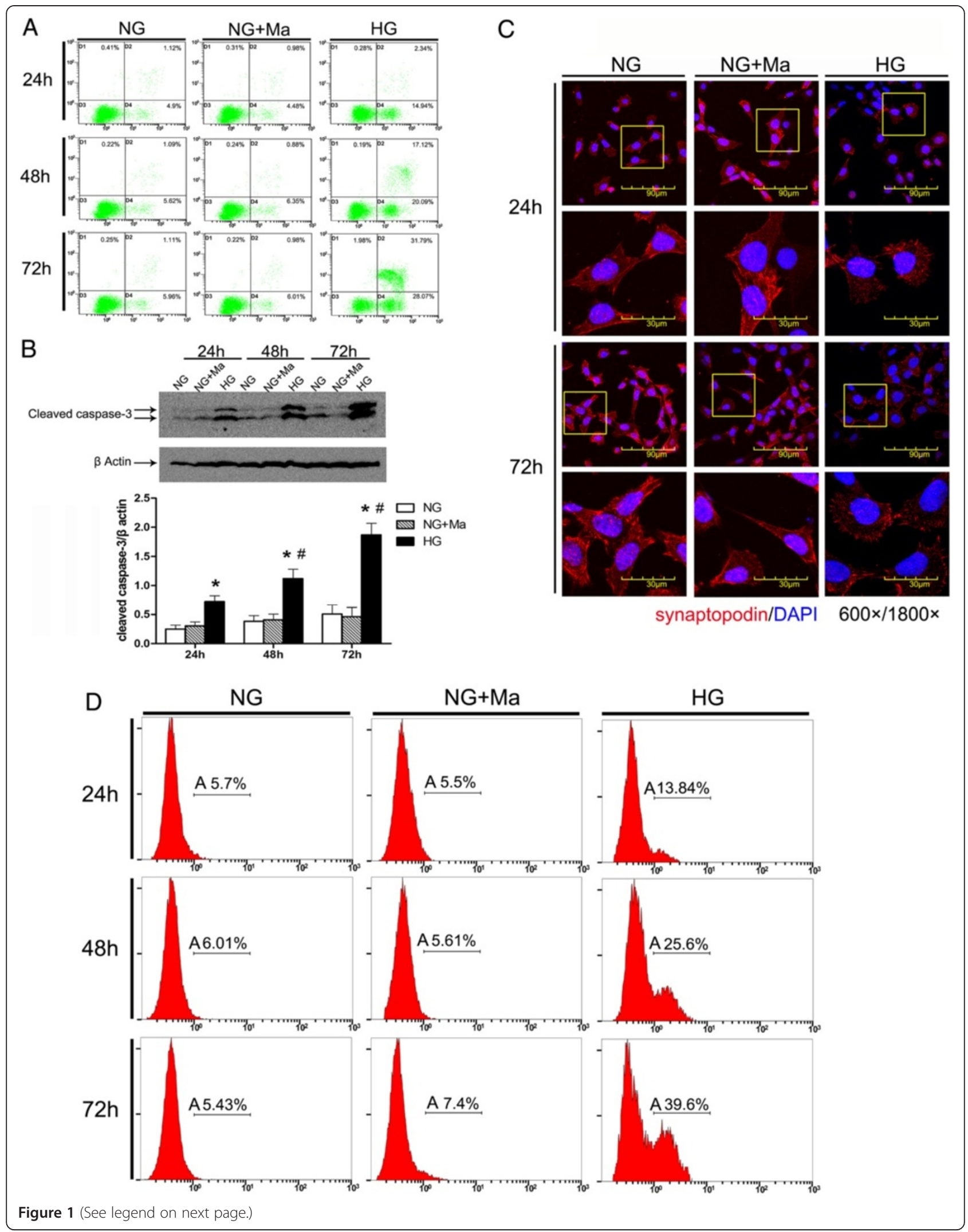




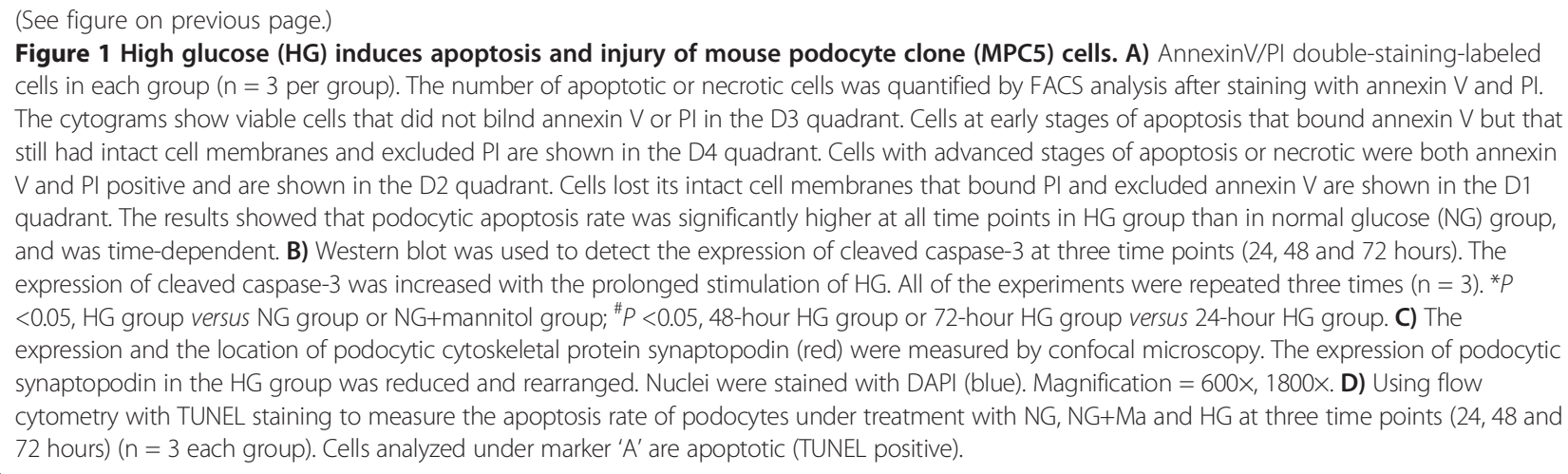

$(0.2 \mathrm{mg} / \mathrm{ml})$ with sterile, deionized water and cryopreserved at $-20^{\circ} \mathrm{C}$.

A solution of rhEGF NtAb (PeproTech, Rocky Hill, NJ, USA) was diluted using the respective $\mathrm{CM}$ to a ratio (EGF: EGF-NtAb) of $1 \mathrm{ng} / \mathrm{ml}: 0.175 \mu \mathrm{g} / \mathrm{ml}$ and was incubated at $37^{\circ} \mathrm{C}$ for one hour before using.

\section{Statistical analysis}

Analysis was done using SPSS 17.0 software. Results are presented as mean \pm standard deviation. One-factor analysis of variance (one-way ANOVA) was used to compare differences among groups. $P<0.05$ was considered statistically significant for all analyses.

\section{Results}

\section{Podocytic apoptosis and injury was induced by HG}

MPC5 cells were cultured in vitro and glucose $(30 \mathrm{mM})$ was added to induce apoptosis to establish a model of podocytic apoptosis and injury. AnnexinV/PI double staining and flow cytometry were used to detect podocytic apoptosis, and the results showed that podocytic apoptosis rates were significantly higher at all time points in the HG group than in the NG group and were time-dependent ( $P$ $<0.05$ ) (Figure 1A). Western blot was used to detect cleaved caspase- 3 . The expression of cleaved caspase- 3 increased more with the prolonged stimulation HG (P $<0.05$ ) (Figure 1B). Confocal immunofluorescence was used to detect the expression of synaptopodin (one of podocytic skelemins), and the results showed that the expression of podocytic synaptopodin in the HG group was reduced and rearranged, while these changes did not occur in the NG+Ma group (Figure 1C). The data suggest that podocytic apoptosis and injury was induced by the increased concentration of glucose, which was aggravated with prolonged stimulation time.

\section{hAd-MSC-CM reduced podocytic apoptosis and injury induced by HG}

After establishing a model of podocytic apoptosis and injury induced by $\mathrm{HG}$ in vitro, we used MSC-CM to culture podocytes in HG. hAd-MSCs can be easily harvested from patients in a simple, minimally invasive lipoaspiration procedure and can be expanded in vitro [20]. Both hAd-MSCs and Wi38 are human fibroblasts, but Wi38-CM does not have the characteristics of hAdMSCs. As a kind of stem cell, hAd-MSCs have the characteristics of self-renewal and multi-differentiation. In addition, hAd-MSCs have a characterized cytokine secretion profile which Wi38 does not. So, we used Wi38$\mathrm{CM}$ as the control [21,22]. Compared with the HG group, hAd-MSC-CM reduced podocytic apoptosis induced by $\mathrm{HG}$ in a dose-dependent manner $(P<0.05)$ (Figure 2A and D), downregulated activated caspase-3 $(P<0.05)$ (Figure $2 \mathrm{~B})$, and prevented the downregulation and rearrangement of synaptopodin (Figure 2C). However, in the Wi38-CM treatment group, there was no significant improvement in these same measures (Figure 2B). Consequently, MSC-CM could prevent podocytic apoptosis induced by HG and reduce the injury of podocytic skelemins, but Wi38-CM could not. Therefore, it was the cytokines present in MSC$\mathrm{CM}$ but not in Wi38-CM that contributed to protecting podocytes from the influence of HG.

\section{Screening of candidate factors in MSC-CM}

We used an antibody-based cytokine array to detect the cytokines in MSC-CM and Wi38-CM and selected the following cytokines with MSC-CM/Wi38-CM >10: basic fibroblast growth factor (bFGF) (3,381-fold), EGF (117fold), IGFBP-1 (45-fold), GDNF (30-fold), and PIGF (123-fold).

It was reported that bFGF could augment podocyte damage, resulting in increased glomerular protein permeability and accelerated glomerulosclerosis [23]. We used ELISA kits to measure the concentration of cytokines in CM: EGF (MSC-CM, $3.81 \pm 0.18 \mathrm{ng} / \mathrm{ml}$; Wi38-CM, $29 \pm 1.1 \mathrm{pg} / \mathrm{ml}$ ), IGFBP-1 (MSC-CM, $0.98 \pm$ $0.01 \mathrm{ng} / \mathrm{ml}$; Wi38-CM, $21.7 \pm 1.9 \mathrm{pg} / \mathrm{ml}$ ), GDNF (MSC$\mathrm{CM}, 3.37 \pm 0.12 \mathrm{ng} / \mathrm{ml}$; Wi38-CM, $119 \pm 21 \mathrm{pg} / \mathrm{ml})$, 
A
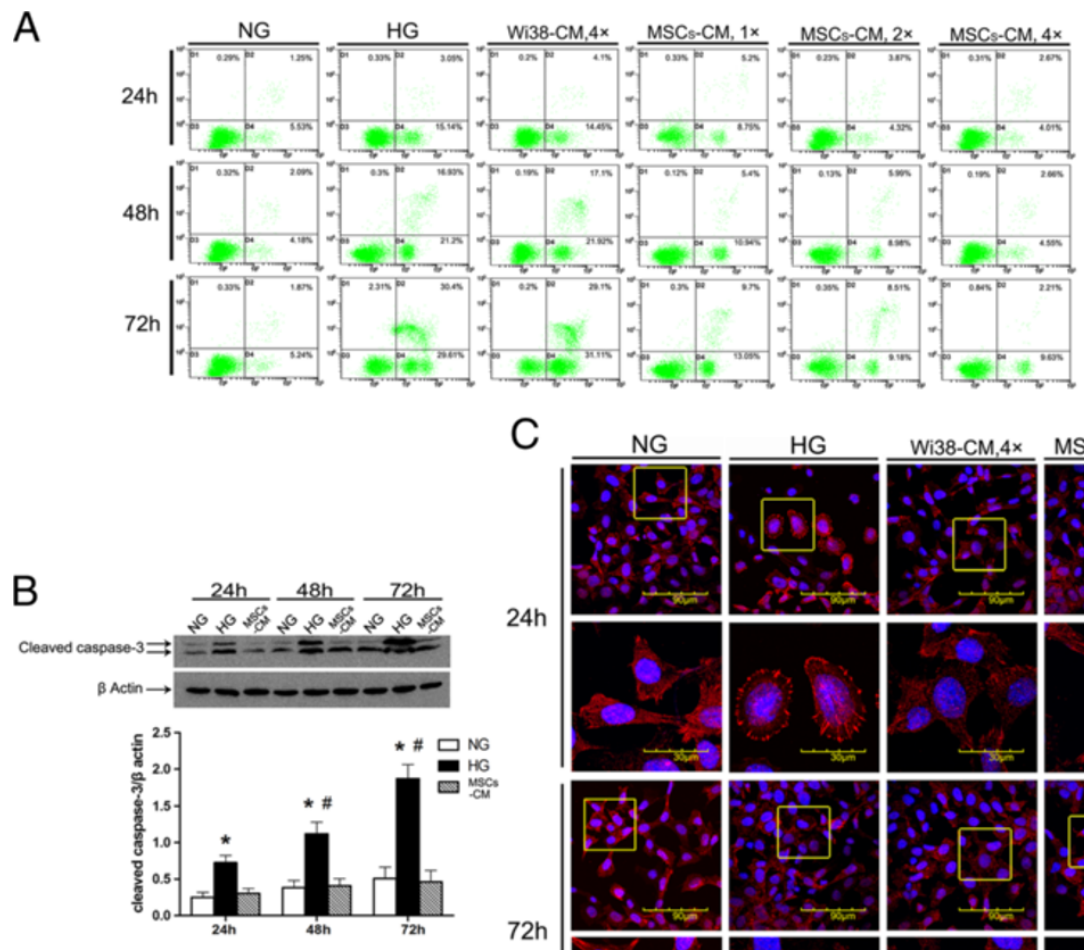

C

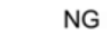

HG Wi38-CM,4x
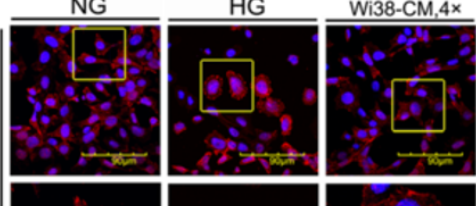

MSCs-CM,4x
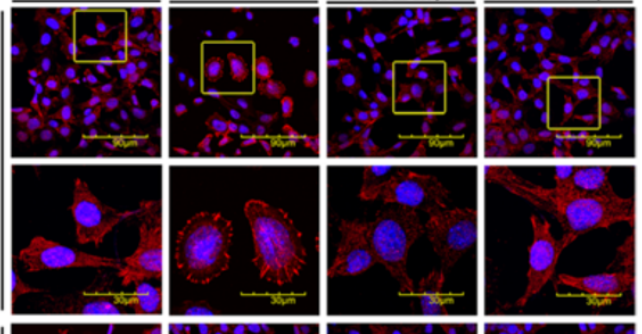

$72 \mathrm{~h}$
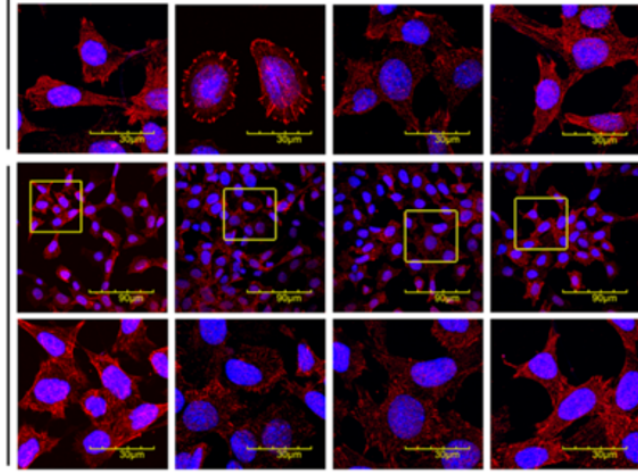

synaptopodin/DAPI $600 \times / 1800 \times$
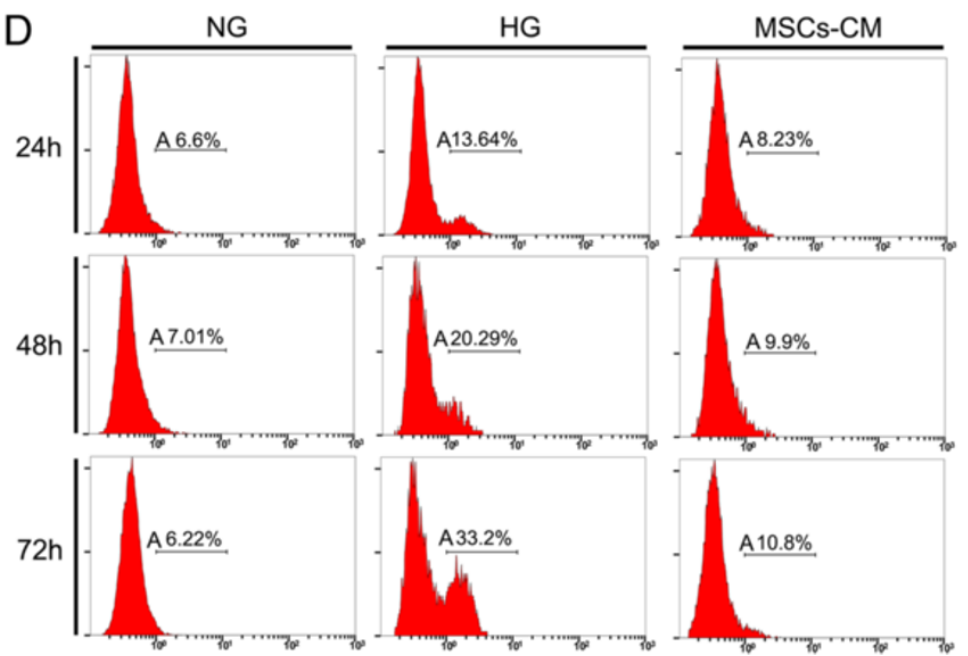

Figure 2 Human adipose-derived mesenchymal stem cells (hAd-MSCs)-conditioned medium (CM) reduces apoptosis and injury of mouse podocyte clone (MPC5) cells induced by high glucose (HG). A) Representative photographs of annexinV/PI double-staining in different groups ( $n=3$ per group) and flow cytometry to test the podocytic apoptosis rate after culture in Wi38-CM or MSC-CM. MSC-CM reduced podocytic apoptosis in a dose-dependent manner, but Wi38-CM did not. B) Western blot was used to detect the expression of cleaved caspase- 3 at three time points (24, 48 and 72 hours). MSC-CM reduced the expression of cleaved caspase-3, but Wi38-CM did not. All of the experiments were repeated three times $(n=3)$. ${ }^{*} P<0.05 \mathrm{HG}$ group versus normal glucose (NG) group or MSC-CM group; \#P $<0.05,48$-hour HG group or 72-hour HG group versus 24-hour HG group. C) The expression and location of the podocytic cytoskeletal protein synaptopodin (red) were measured by confocal microscopy. MSC-CM prevented the downregulation and rearrangement of synaptopodin, but Wi38-CM did not. Nuclei were stained with DAPI (blue). Magnification $=600 \times, 1800 \times$. D) Using flow cytometry with TUNEL staining to measure the apoptosis rate of podocytes under treatment with NG, HG and MSCs-CM at three time points ( 24,48 and 72 hours) ( $n=3$ each group). Cells analyzed under marker ' $A$ ' are apoptotic (TUNEL positive). The results showed that the podocytic apoptosis rate was significantly lower at all time points in the MSCs-CM group than in the HG group. DAPI, 4',6-diamidino-2-phenylindole; PI, propidium iodide. 
and PIGF (MSC-CM, $1.1 \pm 0.09 \mathrm{ng} / \mathrm{ml}$; Wi38-CM, $45.8 \pm 2.2 \mathrm{pg} / \mathrm{ml})$.

Based on these results, we prepared one-component culture medium to podocytes in HG and used flow cytometry to detect podocytic apoptosis. Results showed that $\mathrm{rhEGF}(3.5 \mathrm{ng} / \mathrm{ml})$ effectively inhibited podocytic apoptosis induced by HG $(P<0.05)$ compared with the HG group, but rhIGFBP, rhGDNF, or rhPIGF $(1 \mathrm{ng} / \mathrm{ml}$, $3 \mathrm{ng} / \mathrm{ml}, 1 \mathrm{ng} / \mathrm{ml}$ ) could not (Figure 3C). Therefore, we chose EGF as the candidate cytokine for further investigation (Figure 3).

\section{hAd-MSCs reduce podocytic apoptosis and injury induced} by HG through secreting EGF

To verify that EGF was the effector molecule in CM, we cultured MPC5 cells in the presence of HG plus MSCCM, CM in which EGF was blocked by NtAb, or rhEGF one-component CM. Podocytic apoptosis was detected by annexinV/PI double staining and flow cytometry, cleaved caspase- 3 was detected by Western blot and the expression of synaptopodin and nephrin was detected by confocal immunofluorescence. Compared with the HG group, rhEGF significantly prevented podocytic apoptosis induced by HG as shown by the apoptosis rate $(P<0.05)$ (Figure $4 \mathrm{~A})$ and the expression of cleaved caspase-3 $(P<0.05)$ (Figure $4 \mathrm{~B})$, and reduced podocytic injury as shown by the expression and the arrangement of synaptopodin and nephrin. However, after blocking EGF in MSC-CM with NtAb, the aforementioned therapeutic effect of MSC-CM was significantly decreased $(P<0.05)$ (Figure $4 \mathrm{C}$ and $\mathrm{D})$.

\section{Discussion}

Podocytes not only take part in the formation of the filtration barrier but also express many kinds of protein molecules, including synaptopodin and nephrin. The synaptopodin protein of podocytic skelemins is a marker of podocytic differentiation and maturation, which can maintain the normal morphology of podocytes, the normal structure of the glomerular filtration membrane and

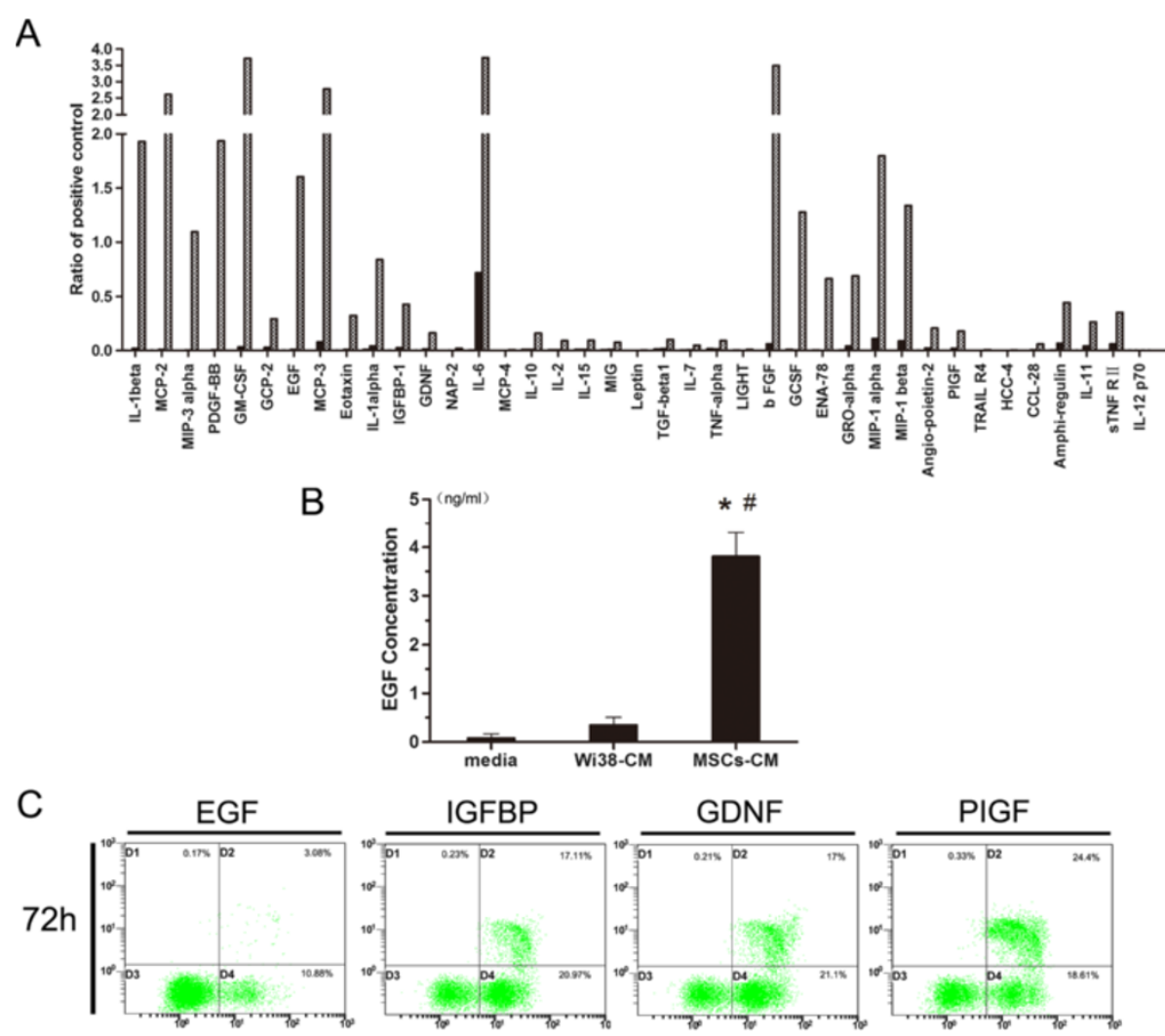

Figure 3 Screening candidate effector factors in human adipose-derived mesenchymal stem cells (hAd-MSCs)-conditioned medium (CM). A) Cytokine microarray to determine the soluble cytokines present in blank medium, Wi38-CM and MSC-CM. Cytokines of MSC-CM/ Wi38-CM >10: basic fibroblast growth factor (bFGF) (3,381-fold), epithelial growth factor (EGF) (117-fold), insulin-like growth factor binding protein (IGFBP)-1 (45-fold) and glial cell line-derived neurotrophic factor (GDNF) (30-fold). The data were normalized to the respective positive controls. B) The concentration of EGF in CM was determined by ELISA. MSC-CM: $3.81 \pm 0.18 \mathrm{ng} / \mathrm{ml}$; Wi38-CM: $29 \pm 1.1 \mathrm{pg} / \mathrm{ml}$. *P $<0.05$ Wi38-CM or MSCCM versus blank medium; \#P <0.05, MSC-CM versus Wi38-CM. C) AnnexinV/PI double staining labeled cells in each group ( $\mathrm{n}=3$ per group) and flow cytometry to determine the podocytic apoptosis rate. Growth factor (EGF) significantly reduced podocytic apoptosis,but other factors (IGFBP, GDNF, PIGF) did not. Pl, propidium iodide. 


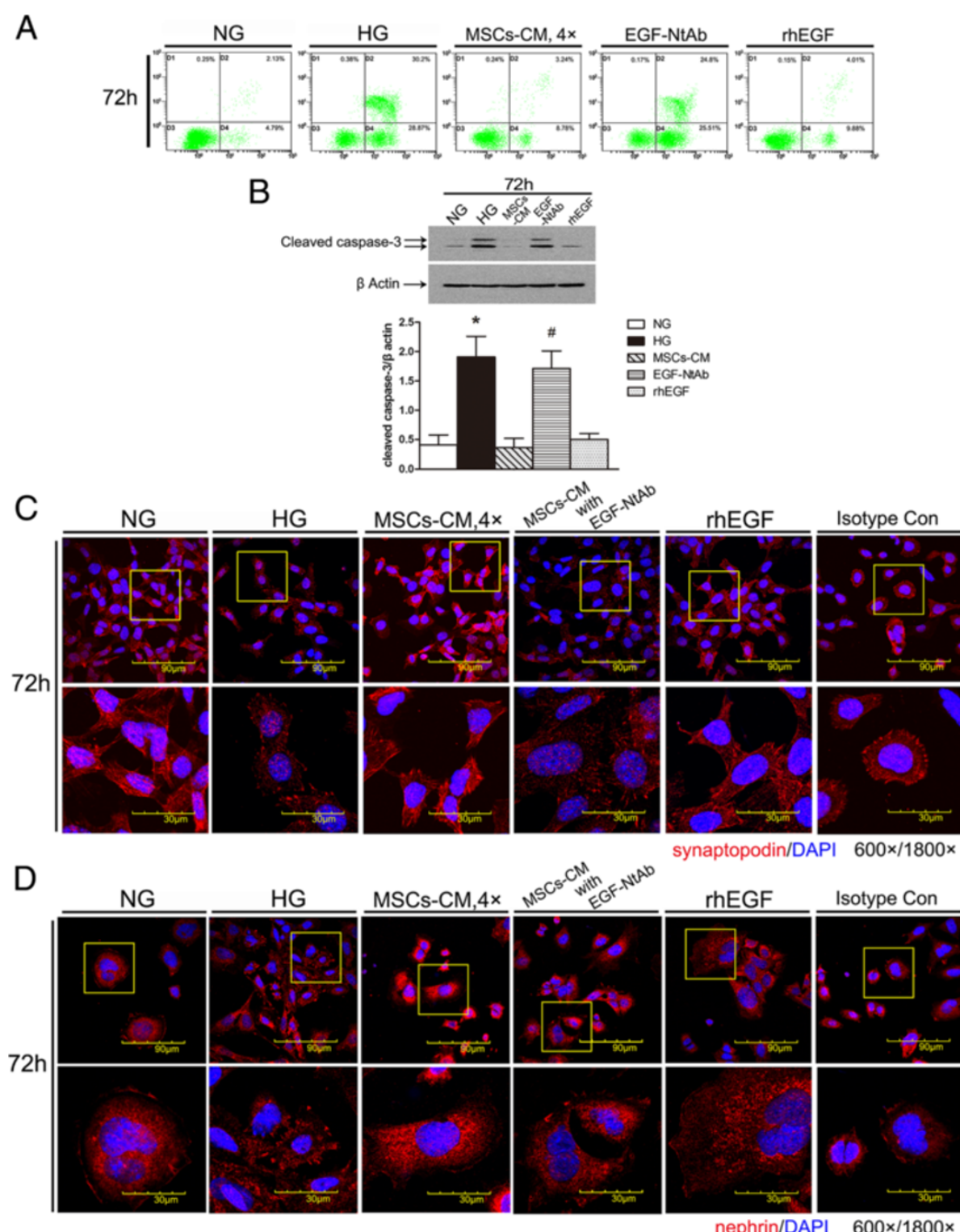

Figure 4 Epithelial growth factor (EGF) is the main factor in human adipose-derived mesenchymal stem cells (hAd-MSCs)-conditioned medium (CM) to reduce apoptosis and injury of mouse podocyte clone (MPC5) cells induced by high glucose (HG). A) AnnexinV/PI double staining labeled cells in each group ( $n=3$ per group) and flow cytometry to determine podocytic apoptosis rate. Recombinant human (rh) EGF group significantly reduced podocytic apoptosis but EGF-neutralizing antibody (NtAb) did not. B) Western blot was used to detect the expression of cleaved caspase-3 at three time points ( 24,48 and 72 hours). rhEGF reduced the expression of cleaved caspase-3, but EGF-NtAb did not. All of the experiments were repeated three times $(n=3) .{ }^{*} P<0.05 \mathrm{HG}$ group versus normal glucose (NG) group, MSC-CM group or rhEGF group; ${ }^{P} P<0.05$, EGF-NtAb versus NG group, MSC-CM group or rhEGF group. C) The expression and the location of the podocytic cytoskeletal protein synaptopodin (red) were measured by confocal microscopy. rhEGF prevented the downregulation and rearrangement of synaptopodin but EGF-NtAb did not. Nuclei were stained with DAPI (blue). Magnification $=600 x, 1800 x$. D) The expression of the podocytic membrane protein nephrin (red) was measured by confocal microscopy. rhEGF prevented the downregulation and disorder of nephrin but EGF-NtAb did not. Nuclei were stained with DAPI (blue). Magnification =600x, 1800 ×. DAPI, 4',6-diamidino-2-phenylindole; PI, propidium iodide.

the charge barrier $[24,25]$. Some studies have suggested a relationship between the occurrence and development of DN proteinuria and podocytic apoptosis and injury $[26,27]$. Nephrin is a single-spanning transmembrane immunoglobulin (Ig) superfamily protein and an integral component of the podocyte slit diaphragm, a structure important to the glomerular selectivity filter [28]. Glucose at high levels could reduce the number and density of podocytes, destroy the integrity of the glomerular filtration membrane and change its selective permeability, 
so as to promote the progression of glomerular sclerosis and accelerate the progress of DN [25,27]. Meanwhile, the loss and injury of interacting proteins of podocytes, such as synaptopodin and nephrin, could activate apoptosis and destroy the integrity of the slit membrane, so as to aggravate proteinuria and accelerate the progression of DN [29]. Consequently, the apoptosis of podocytes and injury of the synaptopodin, nephrin structure play important roles in the occurrence and development of DN. We established an in vitro model of podocytic apoptosis and injury induced by HG. We found that HG induced podocytic apoptosis, decreased the expression of synaptopodin, nephrin and rearranged the structure pattern of synaptopodin. These results are consistent with previous reports $[7,30]$.

Recent reports have demonstrated the capacity of MSCs to repair tissue injuries [31]. MSCs transplantation is considered safe and has been widely tested in clinical trials with encouraging results [32]. Our finding [33] and those of other researchers suggest that the effect of MSCs is mediated mainly by cytoprotective, antiapoptotic, anti-inflammatory factors [34,35], as well as cellular differentiation [36]. In contrast to bone marrow derived-MSCs, Ad-MSCs are more abundant and seem to have several advantages in their differentiation capacity and reparative function [37]. Our previous research found that hAd-MSCs injected via the tail vein reduced kidney injury in a cell localization-independent manner in a type $1 \mathrm{DN}$ rat model. Therefore, we hypothesized that hAd-MSCs may prevent kidney injury through the paracrine action of secreted cytokines. Adding MSCs$\mathrm{CM}$ to the medium of apoptotic podocytes induced by HG, we found the process of apoptosis was inhibited by MSCs-CM, and the decrease and the disorder of podocytic synaptopodin and nephrin were ameliorated by MSCs-CM. It was reported that MSCs can secrete a variety of soluble cytokines to induce the inhibition of apoptosis and repair cell injury, including EGF, GDNF, vascular endothelial growth factor (VEGF), PDGF, IGFBP and IL-x, among others [17,38-40], and these secreted factors can accumulate in CM. It has been reported that MSC-CM has beneficial effects in a variety of tissue repair and cell therapy models, including inhibiting apoptosis and promoting healing and migration [41]. However, we found that WI38 fibroblasts-CM failed to inhibit podocytic apoptosis or injury efficiently. hAd-MSCs are fibroblast-like cells, but Wi38 fibroblasts have no distinct characteristic of stem cells. We speculated that the efficacy of MSCs was mainly due to their 'stem cell characteristics', that is, due to the differences between MSCs-CM and WI38-CM.

We then analyzed the differences between MSCs-CM and WI38-CM using an antibody-based cytokine array. The results showed that the levels of IGFBP, GDNF,
PIGF and EGF in MSCs-CM were significantly higher than those in WI38-CM but only a dose of EGF equivalent to that in CM could inhibit podocytic apoptosis efficiently. Therefore, we chose EGF as the candidate. EGF can promote the repair and regeneration of damaged epidermis, and in both in vitro and in vivo experiments EGF can induce anti-apoptosis and repair the damage of epithelial cells, which was also seen in renal tubular epithelial cells, intestinal epithelial cells, and vascular smooth muscle cells $[39,40,42]$. Whether EGF can inhibit podocytic apoptosis and injury induced by HG has not been reported.

We found that EGF alone could inhibit podocytic apoptosis injury induced by HG but these effects were diminished by blocking EGF with NtAb. We suggest that EGF is necessary for hAD-MSCs to exert their antiapoptosis and anti-injury therapeutic benefits to podocytic apoptosis and injury induced by HG. One study found that inhibition of EGFR induced the apoptosis of intestinal epithelial cells through an EGF receptor (EGFR)/p38 $\alpha /$ mitogen-activated protein kinase (MAPK)/ Bax signaling pathway [42]. In addition, Caraglia et al. reported that EGF inhibited IFN- $\alpha$-induced apoptosis in epidermoid tumors by activating the EGF-dependent Ras/extracellular signal-regulated kinase (Erk) signaling pathway [43]. However, Bollee et al. reported that epidermal growth factor receptor (EGFR) promotes glomerular injury and renal failure in rapidly progressive crescentic glomerulonephritis [44]. Maybe different mechanisms are involved in these studies. Targeting EGF may have benefit in the diseased kidney. Nevertheless, the mechanism through which EGF secreted into the hAd-MSC-CM to prevent podocytic apoptosis and injury induced by HG needs further study.

\section{Conclusions}

Our findings suggest that hAd-MSCs reduce podocytic apoptosis and injury induced by HG, likely by secreting the cytoprotective factor EGF. To our limited knowledge, there has been no report relevant to the effects of MSCs on podocytic injury induced by HG. Our findings may help to develop a new therapeutic method to ameliorate the progress of DN and shed a new light on the mechanisms of the beneficial effects of MSCs on DN.

\section{Abbreviations}

CM: Conditioned medium; DAPI: 4',6-diamidino-2-phenylindole; DN: Diabetic nephropathy; EGF: Epithelial growth factor; EGFR: EGF receptor; ELISA: Enzyme-linked immunosorbent assay; FCS: Fetal calf serum;

GDNF: Glial cell line-derived neurotrophic factor; hAd-MSCs: Human adiposederived MSCs; HG: High glucose; HRP: Horseradish peroxidase; IFN: Inhibited interferon; IGFBP: Insulin-like growth factor binding protein; MPC5: Mouse podocyte clone 5; MSCs: Mesenchymal stem cells; NG: Normal glucose; NG+Ma: Mannitol control; NtAb: Neutralizing antibody; OD: Optical density; one-way ANOVA: One-factor analysis of variance; PBS: Phosphate-buffered saline; PI: Propidium iodide; PIGF: Placental growth factor; rhEGF: Recombinant 
human EGF; RPMI: Roswell Park Memorial Institute; VEGF: Vascular endothelial growth factor; Wi38: Human embryonic lung cells.

\section{Competing interests}

The authors declare that they have no competing interests.

\section{Authors' contributions}

DGL conceived and designed the experiments, performed the experiments, analyzed the data, and wrote the paper. NW performed the experiments, analyzed the data, and wrote the paper. LZ, HYZ and XYB conceived and designed the experiments and analyzed the data. BF, SYC and WGZ performed the experiments, analyzed the data. XFS performed the experiments and revised the paper. RSL and XMC conceived and revised the paper critically for important intellectual content, and gave final approval of the version to be published. All authors read and approved the final manuscript.

\section{Acknowledgements}

Funding: This research was supported by a grant (2011CB964904) from the National Basic Research Program of China, a grant (2011AA020115) from the National High Technology Research and Development Program, a grant (BWS11J027) Military Medical and Techenology 'Twelfth Five-Year Plan' Science and Research Key Program, a grant (61101218) from the National Natural Science Foundation and a grant (2011111) from the New Star Program of Beijing Science and Technology Commission.

Received: 19 December 2012 Revised: 2 August 2013

Accepted: 23 August 2013 Published: 1 September 2013

\section{References}

1. Shimoi A, Hatakeyama H, Koizumi H, Satoh H, Watanabe M: Unchanged distribution density of anionic sites on the glomerular wall in rats with streptozotocin-induced diabetic nephropathy. Toxicol Pathol 2012, 40:789-796.

2. Xu ZG, Ryu DR, Yoo TH, Jung DS, Kim JJ, Kim HJ, Choi HY, Kim JS, Adler SG, Natarajan R, Han DS, Kang SW: P-Cadherin is decreased in diabetic glomeruli and in glucose-stimulated podocytes in vivo and in vitro studies. Nephrol Dial Transplant 2005, 20:524-531.

3. Isermann B, Vinnikov IA, Madhusudhan T, Herzog S, Kashif M, Blautzik J, Corat MA, Zeier M, Blessing E, Oh J, Gerlitz B, Berg DT, Grinnell BW, Chavakis T, Esmon CT, Weiler H, Bierhaus A, Nawroth PP: Activated protein $C$ protects against diabetic nephropathy by inhibiting endothelial and podocyte apoptosis. Nat Med 2007, 13:1349-1358.

4. Wang H, Madhusudhan T, He T, Hummel B, Schmidt S, Vinnikov IA, Shahzad K, Kashif M, Muller-Krebs S, Schwenger V, Bierhaus A, Rudofsky G, Nawroth PP, Isermann B: Low but sustained coagulation activation ameliorates glucose-induced podocyte apoptosis: protective effect of factor V Leiden in diabetic nephropathy. Blood 2011, 117:5231-5242.

5. Griffin SV, Petermann AT, Durvasula RV, Shankland SJ: Podocyte proliferation and differentiation in glomerular disease: role of cell-cycle regulatory proteins. Nephrol Dial Transplant 2003, 18:vi8-vi13.

6. Wang RQ, Nan YM, Wu WJ, Kong LB, Han F, Zhao SX, Kong L, Yu J: Induction of heme oxygenase-1 protects against nutritional fibrosing steatohepatitis in mice. Lipids Health Dis 2011, 10:31.

7. Eid AA, Gorin Y, Fagg BM, Maalouf R, Barnes JL, Block K, Abboud HE: Mechanisms of podocyte injury in diabetes: role of cytochrome P450 and NADPH oxidases. Diabetes 2009, 58:1201-1211.

8. Maxson S, Lopez EA, Yoo D, Danilkovitch-Miagkova A, Leroux MA: Concise review: role of mesenchymal stem cells in wound repair. Stem Cells Trans Med 2012, 1:142-149.

9. Coli A, Nocchi F, Lamanna R, lorio M, Lapi S, Urciuoli P, Scatena F, Giannessi E, Stornelli MR, Passeri S: Isolation and characterization of equine amnion mesenchymal stem cells. Cell Biol Int Rep 2011, 18:e00011.

10. Chase LG, Lakshmipathy U, Solchaga LA, Rao MS, Vemuri MC: A novel serum-free medium for the expansion of human mesenchymal stem cells. Stem Cell Res Ther 2010, 1:8.

11. Schrijvers BF, De Vriese AS, Flyvbjerg A: From hyperglycemia to diabetic kidney disease: the role of metabolic, hemodynamic, intracellular factors and growth factors/cytokines. Endocr Rev 2004, 25:971-1010.

12. Bruno S, Grange C, Deregibus MC, Calogero RA, Saviozzi S, Collino F, Morando L, Busca A, Falda M, Bussolati B, Tetta C, Camussi G: Mesenchymal stem cell-derived microvesicles protect against acute tubular injury. J Am Soc Nephrol 2009, 20:1053-1067.

13. Togel F, Hu Z, Weiss K, Isaac J, Lange C, Westenfelder C: Administered mesenchymal stem cells protect against ischemic acute renal failure through differentiation-independent mechanisms. Am J Physiol Renal Physiol 2005, 289:F31-F42.

14. Burst V, Putsch F, Kubacki T, Volker LA, Bartram MP, Muller RU, Gillis M, Kurschat CE, Grundmann F, Muller-Ehmsen J, Benzing T, Teschner S: Survival and distribution of injected haematopoietic stem cells in acute kidney injury. Nephrol Dial Transplant 2012, 28:1131-1139.

15. Duffield JS, Park KM, Hsiao LL, Kelley VR, Scadden DT, Ichimura T, Bonventre JV: Restoration of tubular epithelial cells during repair of the postischemic kidney occurs independently of bone marrow-derived stem cells. J Clin Invest 2005, 115:1743-1755.

16. Yang Z, Li K, Yan X, Dong F, Zhao C: Amelioration of diabetic retinopathy by engrafted human adipose-derived mesenchymal stem cells in streptozotocin diabetic rats. Graefe's Arch Clin Exp Ophthalmol 2010, 248:1415-1422.

17. Salgado AJ, Reis RL, Sousa NJ, Gimble JM: Adipose tissue derived stem cells secretome: soluble factors and their roles in regenerative medicine. Curr Stem Cell Res Ther 2010, 5:103-110.

18. Danchuk S, Ylostalo JH, Hossain F, Sorge R, Ramsey A, Bonvillain RW, Lasky JA, Bunnell BA, Welsh DA, Prockop DJ, Sullivan DE: Human multipotent stromal cells attenuate lipopolysaccharide-induced acute lung injury in mice via secretion of tumor necrosis factor-alpha-induced protein 6 . Stem Cell Res Ther 2011, 2:27.

19. Susztak K, Raff AC, Schiffer M, Bottinger EP: Glucose-induced reactive oxygen species cause apoptosis of podocytes and podocyte depletion at the onset of diabetic nephropathy. Diabetes 2006, 55:225-233.

20. Cao Y, Sun Z, Liao L, Meng Y, Han Q, Zhao RC: Human adipose tissue-derived stem cells differentiate into endothelial cells in vitro and improve postnatal neovascularization in vivo. Biochem Biophys Res Commun 2005, 332:370-379.

21. Chen L, Tredget EE, Wu PY, Wu Y: Paracrine factors of mesenchymal stem cells recruit macrophages and endothelial lineage cells and enhance wound healing. PloS One 2008, 3:e1886.

22. Ben Azouna N, Jenhani F, Regaya Z, Berraeis L, Ben Othman T, Ducrocq E, Domenech J: Phenotypical and functional characteristics of mesenchymal stem cells from bone marrow: comparison of culture using different media supplemented with human platelet lysate or fetal bovine serum. Stem Cell Res Ther 2012, 3:6.

23. Floege J, Kriz W, Schulze M, Susani M, Kerjaschki D, Mooney A, Couser WG, Koch KM: Basic fibroblast growth factor augments podocyte injury and induces glomerulosclerosis in rats with experimental membranous nephropathy. J Clin Invest 1995, 96:2809-2819.

24. Durvasula RV, Shankland SJ: Podocyte injury and targeting therapy: an update. Curr Opin Nephrol Hypertens 2006, 15:1-7.

25. Yanagida-Asanuma E, Asanuma K, Kim K, Donnelly M, Young Choi H, Hyung Chang J, Suetsugu S, Tomino Y, Takenawa T, Faul C, Mundel P: Synaptopodin protects against proteinuria by disrupting Cdc42:IRSp53: Mena signaling complexes in kidney podocytes. Am J Pathol 2007, 171:415-427.

26. Thomas MC: Pathogenesis and progression of proteinuria. Contrib Nephrol 2011, 170:48-56

27. Sakoda $\mathrm{M}$, Itoh $\mathrm{H}$, Ichihara A: Podocytes as a target of prorenin in diabetes. Curr Diabetes Rev 2011, 7:17-21.

28. Wu F, Saleem MA, Kampik NB, Satchwell TJ, Williamson RC, Blattner SM, Ni L, Toth T, White G, Young MT, Parker MD, Alper SL, Wagner CA, Toye AM: Anion exchanger 1 interacts with nephrin in podocytes. J Am Soc Nephrol 2010, 21:1456-1467.

29. Chen YQ, Wang XX, Yao XM, Zhang DL, Yang XF, Tian SF, Wang NS: MicroRNA195 promotes apoptosis in mouse podocytes via enhanced caspase activity driven by BCL2 insufficiency. Am J Nephrol 2011, 34:549-559.

30. Lee SC, Han SH, Li JJ, Lee SH, Jung DS, Kwak SJ, Kim SH, Kim DK, Yoo TH, Kim JH, Chang SH, Han DS, Kang SW: Induction of heme oxygenase-1 protects against podocyte apoptosis under diabetic conditions. Kidney Int 2009, 76:838-848.

31. Kanazawa H, Fujimoto Y, Teratani T, Iwasaki J, Kasahara N, Negishi K, Tsuruyama T, Uemoto S, Kobayashi E: Bone marrow-derived mesenchymal stem cells ameliorate hepatic ischemia reperfusion injury in a rat model. PLoS One 2011, 6:e19195. 
32. Parekkadan B, Milwid JM: Mesenchymal stem cells as therapeutics. Annu Rev Biomed Eng 2010, 12:87-117.

33. Wang N, Li Q, Zhang L, Lin H, Hu J, Li D, Shi S, Cui S, Zhou J, Ji J, Wan J, Cai G, Chen X: Mesenchymal stem cells attenuate peritoneal injury through secretion of TSG-6. PloS One 2012, 7:e43768.

34. Gnecchi M, He H, Liang OD, Melo LG, Morello F, Mu H, Noiseux N, Zhang L, Pratt RE, Ingwall JS, Dzau VJ: Paracrine action accounts for marked protection of ischemic heart by Akt-modified mesenchymal stem cells. Nat Med 2005, 11:367-368.

35. Mias C, Lairez O, Trouche E, Roncalli J, Calise D, Seguelas MH, Ordener C, Piercecchi-Marti MD, Auge N, Salvayre AN, Bourin P, Parini A, Cussac D: Mesenchymal stem cells promote matrix metalloproteinase secretion by cardiac fibroblasts and reduce cardiac ventricular fibrosis after myocardial infarction. Stem Cells 2009, 27:2734-2743.

36. Yoon BS, Moon JH, Jun EK, Kim J, Maeng I, Kim JS, Lee JH, Baik CS, Kim A, Cho KS, Lee HH, Whang KY, You S: Secretory profiles and wound healing effects of human amniotic fluid-derived mesenchymal stem cells. Stem Cells Dev 2010, 19:887-902.

37. Hong HS, Kim YH, Son Y: Perspectives on mesenchymal stem cells: tissue repair, immune modulation, and tumor homing. Arch Pharm Res 2012, 35:201-211.

38. Bailey AM, Kapur S, Katz AJ: Characterization of adipose-derived stem cells: an update. Curr Stem Cell Res Ther 2010, 5:95-102.

39. Lu C, Ren W, Su XM, Chen JQ, Wu SH, Zhou GP: EGF-recruited JunD/c-fos complexes activate CD2AP gene promoter and suppress apoptosis in renal tubular epithelial cells. Gene 2009, 433:56-64.

40. Ying $W Z$, Zhang $H G$, Sanders PW: EGF receptor activity modulates apoptosis induced by inhibition of the proteasome of vascular smooth muscle cells. J Am Soc Nephrol 2007, 18:131-142.

41. Yew TL, Hung YT, Li HY, Chen HW, Chen LL, Tsai KS, Chiou SH, Chao KC, Huang TF, Chen HL, Hung SC: Enhancement of wound healing by human multipotent stromal cell conditioned medium: the paracrine factors and p38 MAPK activation. Cell Transplant 2011, 20:693-706.

42. Sheng G, Guo J, Warner BW: Epidermal growth factor receptor signaling modulates apoptosis via p38alpha MAPK-dependent activation of Bax in intestinal epithelial cells. Am J Physiol Gastrointest Liver Physiol 2007. 293:G599-G606.

43. Caraglia M, Tagliaferri P, Marra M, Giuberti G, Budillon A, Gennaro ED, Pepe S, Vitale G, Improta S, Tassone P, Venuta S, Bianco AR, Abbruzzese A: EGF activates an inducible survival response via the RAS- > Erk-1/2 pathway to counteract interferon-alpha-mediated apoptosis in epidermoid cancer cells. Cell Death Differ 2003, 10:218-229.

44. Bollee G, Flamant M, Schordan S, Fligny C, Rumpel E, Milon M, Schordan E, Sabaa N, Vandermeersch S, Galaup A, Rodenas A, Casal I, Sunnarborg SW, Salant DJ, Kopp JB, Threadgill DW, Quaggin SE, Dussaule JC, Germain S, Mesnard L, Endlich K, Boucheix C, Belenfant X, Callard P, Endlich N, Tharaux PL: Epidermal growth factor receptor promotes glomerular injury and renal failure in rapidly progressive crescentic glomerulonephritis. Nat Med 2011, 17:1242-1250.

\section{Submit your next manuscript to BioMed Central and take full advantage of:}

- Convenient online submission

- Thorough peer review

- No space constraints or color figure charges

- Immediate publication on acceptance

- Inclusion in PubMed, CAS, Scopus and Google Scholar

- Research which is freely available for redistribution

Submit your manuscript at www.biomedcentral.com/submit
C Biomed Central 